\title{
Das Hochschulrechenzentrum an der TFH Wildau als Dienstleister für Forschung, Lehre und Verwaltung
}

\author{
Dipl.-Ing. Günter Schubert, Dipl.-Ing. Norbert Miersch
}

\section{Einführung}

Begriffe wie Internet, Intranet, Multimedia, Online-Banking, World Wide Web (WWW), Hyperlinks, Surfen im Internet, E-Mail usw. findet man sowohl in einschlägiger Fachliteratur als auch in Tageszeitungen, in Zeitschriften, im Fernsehen usw. Wir befinden uns auf dem Weg von der Industrie- zur Informationsgesellschaft. Alle Bereiche, vor allem Wissenschaft, Wirtschaft, Politik, aber auch soziale und kommunikative Bereiche der Menschen werden von den neuen Informations- und Kommunikationstechnologien beeinflußt. Gewinnung, Speicherung, Verarbeitung, Vermittlung, Verbreitung und Nutzung von Informationen und Wissen sowie die interaktive Kommunikation sind in der Informationsgesellschaft entscheidende Faktoren. Der zeitliche Verlauf dieser Entwicklung wird u.a. maßgeblich vom Aufbau leistungsstarker „Datenautobahnen / Informations-Highways“ bestimmt.

Die Informationsgesellschaft wird unsere bisherigen Arbeits- und Lebensgewohnheiten in einer Weise beeinflussen, die viele vielleicht für Utopie halten. Die fortschreitende Vernetzung läßt räumliche und zeitliche Grenzen unserer Welt vergessen. Wer konnte sich schon vor 5 Jahren vorstellen, daß ein Team uiber Kontinente verteilt, zeitgleich bei einem gemeinsamen Projekt auf die gleichen aktuellen Daten zugreifen kann?

Der Computer und die weltweite Vernetzung durch das Internet sind aus unserem täglichen Leben nicht mehr wegzudenken. Auch in vielen Privathaushalten hat der PC Einzug gehalten. Zahlreiche Provider bieten auch für Privatkunden den Internetzugang an. Dabei nimmt Deutschland durchaus keine Spitzenposition ein. In Finnland haben bereits rund 60 Prozent der Bevölkerung einen Internet-Zugang. Das ist Weltspitze. In den USA sind es 16 Prozent, in Japan 18 Prozent der Bevölkerung. Über die weltweite Anzahl der Nutzer im Internet gibt es keine verläßlichen Zahlen. Geschätzt werden zwischen 23,5 und 35 Millionen Internetzugänge. Prognostiziert werden für das Jahr 2000 über 250 Millionen Internet-Nutzer. Studien haben ergeben, der Anteil der Studenten an der Anzahl der Nutzer liegt bei rund 30 Prozent! Daß das Interesse der Studierenden am Internet-Zugang sehr groß ist, kann aus der Sicht des Hochschulrechenzentrums nur bestätigt werden.

\section{Die Aufgaben des Hochschulrechen- zentrums}

An einer Fachhochschule, wie der Technischen Fachhochschule Wildau, nimmt das Hochschulrechenzentrum einen wichtigen Platz u. a. bei der Bereitstellung kommunikativer Dienste ein.
Das Hochschulrechenzentrum (HRZ), als zentrale Einrichtung der TFH, leitet seine Aufgaben sowohl von den Anforderungen der Forschung und Lehre, der Hochschulverwaltung als auch aus den allgemeinen Anforderungen, die aus der technischen Weiterentwicklung resultieren, ab.

Die sich daraus ergebenen Teilaufgaben sind folgende:

- Bereitstellen von Rechnerkapazitäten im HRZ

- Sicherung eines stabilen Betriebes in den PC- und Workstationlaboren des HRZ

- Wartung, Netzwerkmanagement, Softwareinstallation in den Laboren

- Verwaltung und Verteilung von Software-Lizenzen (für gesamte TFH)

- Führen der Datenbank iiber Ausstattung der TFH an Hard- und Software

- Zuarbeit und Beratung bei Hard- und Software-Beschaffungen

- Betreuung der Nutzer, Hard- und Software in den zentralen Abteilungen und Einrichtungen der Hochschule

- Operative Arbeit (Hilfe bei Störungen an der Rechentechnik im Verwaltungsbereich)

- Netzwerkmanagement des Campusnetzes

- Verwaltung von IP-Nummern

- Betreuung des Zuganges zum Wissenschaftsnetz (WiN)

- Nutzerverwaltung für den Internetzugang (Zuweisung von E-Mail-Adressen)

- Betreuung der WWW-, FTP-, Mail-Server

- Konzeptionelle Arbeit

\section{Die Labore des Hochschulrechenzentrums}

Zur Erfuillung der o.g. Aufgaben stehen u.a. zwei reichhaltig ausgestattete PC-Labore im Hochschulrechenzentrum zur Verfuigung. An insgesamt 36 PC-Arbeitsplätzen können zeitgleich Übungen durchgeführt werden. Anspruchsvolle Software, wie das Datenbanksystem Oracle oder das Programm Mathematica wird in der Ausbildung der Studenten eingesetzt. Scanner, Laserdrucker sowie ein Behindertenarbeitsplatz für Sehgeschädigte vervollständigen die Ausstattung der PCLabore. Der Behindertenarbeitsplatz ist mit einer BrailleZeile versehen, die es auch dem Blinden ermöglicht, den Bildschirminhalt in der fuir ihn lesbaren Schrift zu erfassen. Spezielle Software gestattet es, auch die grafischorientierte Windowsoberfläche dem Blinden zugänglich zu machen. Auf einem in einer Schallschutzhaube untergebrachten Brailledrucker können alpha-numerische Zeichen in Brailleschrift für den Blinden gedruckt werden. „Normale“ Textdokumente werden uiber einen Scanner in Verbindung mit einer OCR-Software eingelesen und iiber die Braillezeile dargestellt. 
Alle Computer des Labores sind an der strukturierten Verkabelung des neuen Campusnetzes angeschlossen und laufen unter dem Netzwerkbetriebssystem IntraNetware. Somit ist von jedem PC-Arbeitsplatz aus der Internetzugang möglich.

Das Workstationlabor ist sowohl fuir den Internetzugang als auch für die Nutzung anspruchsvoller CAD/CAM und FEM-Programme vorgesehen. Mit Hilfe des CAD/CAMProgramms Pro/Engineer ist u.a. das parametrische Konstruieren in 3D möglich. Das erzeugte Modell kann schattiert von allen Seiten durch dynamisches Bewegen im Raum betrachtet werden. Zahlreiche Module ergänzen die Funktionalität und machen das Programm zu einem leistungsstarken Werkzeug des Ingenieurs. Die Berechnung von Spannungen und Verschiebungen an komplizierten Bauteilen sowie die Berechnung statisch unbestimmter Systeme, die sich der manuellen Berechnung entziehen, kann mittels des FEM-Programms COSMOS/M näherungsweise durchgefuihrt werden.

Über Videokonferenzsysteme im ISDN-Labor können Partner miteinander kommunizieren. Sie können hierbei miteinander sprechen, sich gegenseitig sehen und auch durch ein Applikationsharing gemeinsam an einer Anwendung, wie z.B. einem Word-Dokument arbeiten.

\section{Das ATM-Backbone-Netz}

\subsection{Allgemeines}

Um den Kommunikationsanforderungen der Zukunft gerecht zu werden, wird an der TFH-Wildau ein ATM Datennetz errichtet. Im Rahmen der Umgestaltung und territorialen Erweiterung der TFH war die Entscheidung naheliegend, ein Netz auf der "grünen Wiese" aufzubauen. Die territoriale Struktur umfaßt jetzt den alten Campus, die Friedrich-Engels-Straße 63, sowie Teile des Südgeländes des ehemaligen Schwermaschinenbaues, darunter auch Haus 13 in der Bahnhofstraße 1. In dem Haus 13, wo neben der Verwaltung auch das Hochschulrechenzentrum ansässig ist, wurde bereits mit dem Aufbau des neuen Campusnetzes begonnen. In naher Zukunft werden nach der kompletten Vernetzung des Haus 13 schrittweise alle TFH-Einrichtungen in das Campusnetz integriert. Durch einen ATM Backbone werden die einzelnen territorial versetzten Campusteile miteinander verbunden. Vorhandene kleine LANs der Fachbereiche werden nach und nach in das "große Netz" eingebunden. In den vorhandenen LANs selbst müssen neben organisatorischen Veränderungen, Topologieveränderungen, Protokollveränderungen und Softwareveränderungen vorgenommen werden.

Die Notwendigkeit zur Errichtung eines neuen Campusnetzes begriindet sich aus den zur Zeit vorhandenen, grundsätzlichen Anforderungen an das Netz:

- Bedarf, Ressourcen „online“ zwischen Rechnerarbeitsplätzen auszutauschen.

- Rechner- und standortunabhängige Abforderungen von gleichen Informationen.

- Nutzung des Weitverkehrsnetzes durch WWW, e-mail, FTP, Telnet, News.
- Standortunabhängiger Zugriff auf eigenes Homeverzeichnis beispielsweise zum Einbringen von eigenen Homepages (Mitarbeiter, Studenten) oder hochschulspezifischen Informationen mit Internetwerkzeugen.

- Nutzung von ISDN / später B-ISDN.

- Durchfürung von Videokonferenzen im Gesamtcampus oder zwischen der TFH und anderen Institutionen (Unternehmen, andere Bildungseinrichtungen etc.).

- Möglichkeit für Mitarbeiter und Studenten das LAN der Technischen Fachhochschule durch Einwahl iiber Modem zu nutzen, um damit Zugang zum Internet zu erhalten.

- Einbindung eines zuverlässigen Sicherheitskonzeptes muß möglich sein (LAN Security).

Weitere Anforderungen sind:

- Die Einbindung von heterogenen LANs, d. h. Protokolle wie IP, IPX, NetBEUI muissen weiterhin bestehen können.

- „Classes of Service“ für Videokonferenzschaltungen. Möglichkeit der Bandbreitenbiindelung muß gegeben sein.

- Neben dem Ausfuihren von Videokonferenzen und der Kopplung von LANs müssen auch TK-Anlagen miteinander kommunizieren können.

\subsection{Netzwerkkonzeption, passive Netzwerkverkabe- lung}

Die Basis für das neue Campusnetz bildet die passive Netzwerkverkabelung, die zu einem großen Teil bereits im Haus 13 realisiert wurde. Jedes Gebäude der TFH bekommt sukzessive auch eine neue Netzverkabelung. Das passive Netz ist für ein Gebäude hierarchisch aufgebaut und besteht aus folgenden Bestandteilen:

- Primäres LAN (Backbone Verkabelung)

Hierbei handelt es sich um eine Gebäude-zu-Gebäude-Verkabelung, d. h. die primäre Verkabelung soll meist im Keller alle Gebäude auf dem Campusgelände durchlaufen. Der Backbone der TFH umfaßt bisher eine Station. Er endet und beginnt im Hauptwiringcenter des Hauses 13 im Keller. Wie die Backboneverkabelung zwischen Friedrich-Engels-Straße 63 und dem Haus 13 in der Bahnhofstraße 1 gestaltet wird, ist $\mathrm{z}$. Zt. noch nicht festgelegt. Als favorisierte Varianten gelten LWL oder Richtfunk. Es muß immerhin eine Entfernung von $2-3 \mathrm{~km}$ überbrückt werden.

- Sekundäres LAN

Diese Verkabelung wird vom Backbone bis in die letzte Etage des jeweiligen Gebäudes verlegt. Im Haus 13 entstanden hier ein Wiringcenter für das Erdgeschoß und zwei Wiringcenter im 1. Obergeschoß. Als Verkablungsarten sind hier Kupfer und LWL realisiert. In den Wiringcentren der Etagen ist es möglich, Verbindungen zu den Netzwerkdosen der Räume, zum Rangierfeld in der TK-Anlage, aber auch zu anderen Wiringcentren und zu den eingebauten aktiven Komponenten herzustellen. Solche Verbindungen werden manuell mit Patchkabeln hergestellt.

- Tertiäres LAN

Es handelt sich hier um die Verteilung in den einzelnen Etagen. Als Verkabelungsarten sind hier gleicher- 
maßen Kupfer und LWL realisiert. Die meisten Räume des Hauses 13 sind dazu bereits mit einem neuen Kabelkanal und den dazugehörigen Dosen für 10BaseT, aber auch 10Base-FL ausgeriistet.

\subsection{ATM Netzwerk, aktive Komponenten}

Die aktiven Komponenten unterstiitzen ATM und Ethernet. Je nach Komponente arbeiten sie im Layer 1 bis 4 des OSI-7 Schichtmodelles und sind 3 Com-Produkte. Im Haus 13 werden bisher folgende Komponenten eingesetzt:

FMS II (Flexibel Media Stack)

Es handelt sich hier um einen 12 Port Repeater für 10Base-T, sowie einen 13. Port für 10Base-FL oder AUI. Jeder Repaeter besitzt ein Managementmodul. Dieses Modul sammelt SNMP Variable, die an der Managementstation mittels RMON ausgewertet werden. Jeder Repeater hat außerdem eine IP-Adresse, mit der er dem jeweiligen IP-Netz (IP-Subnetz) zugeordnet ist. Auf das Managementmodul kann neben dem Zugriff uiber SNMP auch iiber Telnet zugegriffen werden. Außerdem ist durch ein RMON-Tool ein Capturing möglich. Der Repeater hat folgende Zusatzfunktionen:

- Resilient Links:

Ist der Link mit einem im Netz integrierten Rechner bzw. Drucker gegeben, leuchtet für den jeweiligen Port eine Linklampe. Das Verarbeiten von Datenpaketen für den gesamten Repeater wird mit einer gelben Paketlampe angezeigt. Repeater untereinander sind auch kaskadierbar. Das kann mittels 10Base-T an Port 12 und Port 1 der jeweiligen Repeater ermöglicht werden. Jedoch ist hierfür ein Schalter, der das Vertauschen von $\mathrm{Rx}$ und Tx verhindert, zu betätigen.

- LAN-Security:

Bei der NTK (need to know) Funktion, wird pro „Dedicated“" Port nur eine Sendeadresse (MAC-Adresse) zugelassen. Bei der DUD- (disable unauthorization device) Funktion werden für einen „Secure“ Port nur autorisierte MAC-Adressen bearbeitet (maximal 34). Pakete nicht autorisierter Adressen werden verworfen. Die vom Netzwerk-Administrator per Konfiguration vergebene Autorisierung für Sende- und Empfängeradressen kann nicht iiberschrieben werden. Etwaige Sendeversuche nichtautorisierter Netzwerkteilnehmer werden unmittelbar der Managementkonsole gemeldet (Trap). - RMON:

Remote Monitoring ist durch Managementmodul möglich. Die Auswertung ist durch ein Softwarezusatzprodukt „Transcend LAN Sentry“ der Firma 3 Com möglich.

\section{Cellplex 7000 / SuperStack II-Switch 2700}

Der Cellplex 7000 existiert zur Zeit mit 3 ATM Cellswitchmodulen (CP 7200) im Erdgeschoß und 2 ATM Cellswitchmodulen (CP 7200) in einem Wiringcenter des 1. Obergeschosses im Haus 13. Das zweite Wiringcenter im 1. Obergeschoß wurde zur Zeit mit nur einem SuperStack II-Switch 2700 ausgestattet.

Die Cellplex 7200 Module besitzen jeweils 3 ATM10Base-FL-Ports für direkt $155 \mathrm{Mbits} / \mathrm{s}$ und $12 \mathrm{ATM}$-ToEthernet-10Base-T-Ports für $10 \mathrm{Mbits} / \mathrm{s}$, an dem jeweils ein „elan“ (LEC) angebunden werden kann. Hinweis: Bei 3 Com werden virtuelle LANs als „elans“ bezeichnet. An einen ATM-To-Ethernetport kann entweder direkt ein Ethernetadapter oder ein FMS II angeschlossen werden. Der Switch 2700 besitzt nur 1 ATM-Port und 12 ATM-ToEthernetports. Alle Switche besitzen außerdem Managementports, Serviceports und Controlterminalports. Die Verbindung zwischen Rechner oder Drucker und einem ATM-Switchport wird als UNI (User-To-Network-Interface) bezeichnet. Hier wird der 5 Octetkopf der 53 Octet großen ATM-Zelle durch ein Generic Flow Control Code (GFC) ergänzt. Die Verbindung zwischen 2 Switchen wird als NNI (Network-To-Network-Interface) bezeichnet. Der GFC fehlt hier. Die ATM-Zelle bleibt auch hier 53 Octets groß. Wie beim FMS II ist ein Direktzugriff uiber Telnet und über SNMP möglich. Vorhandene Links sowie der Datenverkehr werden über Lampen signalisiert.

Die Switche unterstützen die Bildung von virtuellen LANs. Für die TFH-Wildau wurden 16 elans gebildet (16VPorts). Die Definition derartiger logischer Arbeitsgruppen erfolgt standort- und topologieunabhängig durch Zuweisung der Ethernet-Ports am Switch-Modul zu dem „elan“ per Software. Per Software wird auch am Cellplex die LAN Emulation konfiguriert.

\section{NBII (Netbuilder II)}

Bei dem ATM-Netbuilder II handelt es sich um einen Backbonerouter, der IP-Netze und IPX-Netze routen kann. Der Router ist mit den Cellplexen verbunden und so konfiguriert, das er zur Zeit 16 „elans“ (Vports) routet. Um dem geplanten Sicherheitskonzept Rechnung zu tragen, bei dem das Managementnetz ein eigenständiges IP-Netz bilden muß, wurden die für die TFH vergebenen CLASS-C Netze noch einmal gesubnetet. Jeder Fachbereich bzw. Labor erhält somit ein eigenständiges IP-Netz bzw. IP-Subnetz. Der Router muß aber insgesamt 21 Netze routen. Hier gab es hinsichtlich der Software noch Probleme, die mit einem neuen Softwarestand gelöst werden sollen. Der NBII hat eine HD sowie ein 3,5“ Floppy, so daß er auch uiber Diskette gebootet werden kann. Wie beim FMSII und den Cellplexen/Linkswitchen ist ein Zugriff mittels Telnet oder SNMP möglich. Ein Managementport, Serviceport und ein Controlterminalport gehören mit zur Ausstattung. Bild 1 stellt die 1. Ausbaustufe des ATM-Backbone-Netzes in Haus 13 vereinfacht dar.

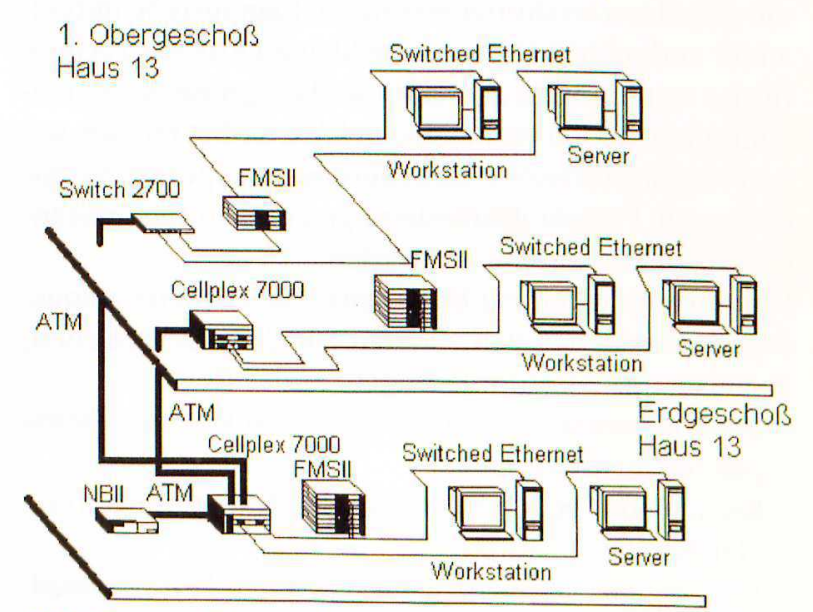

Bild 1: Ethernet/ATM, TFH Wildau 


\subsection{ATM LAN Emulation}

Für das ATM Netz der TFH Wildau ist das LAN Emulationsverfahren konfiguriert worden.

„Die LAN Emulation (LE) erlaubt eine protokolltransparente Kopplung von herkömmlichen LANs über ATM sowie die Kommunikation mit direkt an ATM angeschlossenen Endgeräten. LE gaukelt der herkömmlichen LAN-Umgebung vor, es handele sich bei ATM nur um ein weiteres 802.x-LAN, d. h. alle ATM-Eigenschaften werden nach oben hin versteckt. Hierdurch können alle vorhandenen Endgeräte und Applikationen ohne Modifikation weiter genutzt werden." /1/

Füir die LAN Emulation laufen komplexe Vorgänge in den aktiven Komponenten (Switche, Router) ab. Für das ATM-Netz der TFH Wildau wurde ein LECS (LAN Emulation Configuration Server), 16 LES / BUS (LAN Emulation Server/Broadcast/Unknown Server), und entsprechende dem Änderungsdienst vorbehaltene LEC (LAN Emulation Clients) konfiguriert. Als LECs sind die ATM-To-EthernetSwitschports konfiguriert.

\subsection{Netzwerkmanagement}

Das Netzwerkmanagement wird in erster Linie manuell von der Managementstation durchgeführt. Die TFH Wildau hat dafuir eine SUN SPARC-Station M5 mit 64 Mbyte RAM. Über das SNMP (Simple Network Management Protokoll) und eine MIB (Management Information Base) werden Managementinformationen verwaltet und ausgewertet. In jeder aktiven Komponente des Netzwerkes befindet sich, wie o.g. ein Managementmodul (Agent), das Werte den SNMP Variablen zuweist. Diese SNMP-Variablen werden in der MIB der Managementstation verwaltet und ausgewertet. Aktive Bauteile, wie Netzwerkkarten in Rechnern oder Druckern können für die MIB anhand der MAC Adresse, die einen herstellerspezifischen Vendorcode beinhaltet, erkannt werden. Durch die Nutzung der Software „HP Open ViewNetwork Node Manager" und den „Transcsend-Enterprise Manager V 4.0 for Unix" (RMON) kann ein komplettes Management durchgefuihrt werden.

Die Root der Managementsoftware ist folgendermaßen untergliedert:

- IP-Internet:

Hier werden alle IP-Netze, die durch den Netbuilder II „zusammengeroutet“ werden, dargestellt. Durch Anklicken der einzelnen Symbole kann immer tiefer bis zu den einzelnen Segmenten, Rechnern, aktiven Bauteilen verzweigt werden. Über Grafiken und Statistiken kann sogar für jeden Port eines aktiven Bauteils eine Analyse gemacht werden. Die CLASS-C IPNetze der TFH - Wildau sind zur Zeit in 21 IP-Netze und Subnetze unterteilt, wobei das Managementnetz ein eigenständiges Netz ist.

- ATM-Devices:

Die grafische Darstellung aller aktiven ATM Komponenten mit ihren Verbindungen wird hier erzeugt.

- Virtual LANs:

Hier erfolgt die Darstellung aller elans und des gesamten LESystems (LECS,LES/BUS,LEC) und der
LANedges (Switche). Hier können auch die elans verschoben werden (Zuweisung eines elans für einen Switchport).

- ATM-Network:

In diesem graphischen Untermenui werden die Verbindungen der aktiven ATM-Bauelemente untereinander dargestellt.

Besonderheiten :

Hinter jedem ICON eines aktiven Bauteiles kann man eine Managementfunktion hinterlegen. Beispielsweise ist es möglich, mit dem Netbuildersymbol ein Telnet, oder mit dem Repeatersymbol die grafische Darstellung des Repeaters mit all seinen Ports zu verknuipfen. Eine weitere Besonderheit ist das Trap. Hier wird der Netzwerkadministrator bei bestimmten Ereignissen gewarnt. Siehe oben FMS II. Als letztes sei hier noch das Capturing genannt. Hier können Frames mitgeschrieben werden.

\section{Zur Internet-Konnektivitäł}

Die TFH Wildau nutzt iiber den DFN-Verein (Verein zur Förderung eines deutschen Forschungsnetzes) den Zugang zum Internet. Dazu steht eine Datex-P-Standleitung von Wildau nach Berlin mit einer Kapazität von 64 kB/s zur Verfügung. Über den Gateway-Rechner des DFN-Vereins in Berlin erfolgt der Zugang über das Wissenschaftsnetz zum Internet. Zur Zeit wird durch den DFN-Verein ein leistungsstarkes Breitband-Wissenschaftsnetz (B-WiN) aufgebaut, das Übertragungsraten von $155 \mathrm{Mbit} / \mathrm{s}$ zuläßt. Damit wird es neben einer schnelleren Übertragung der Daten und bisherigen Dienste auch möglich sein, Sprache zu übertragen. Gegenwärtig wird vom DFN-Verein an einem Konzept gearbeitet, für die Nutzer des B-WiN auch Telefoniedienste uiber das Netz zur Verfuigung zu stellen.

Die TFH Wildau wird im Juli 1997 uiber das MAN Potsdam Zugang zum B-WiN erhalten (MAN= MetropolitanAreaNetwork). Geplant ist zunächst ein $2 \mathrm{MB} / \mathrm{s}$-Verbindung zwischen Wildau und Potsdam, die auf $34 \mathrm{MB}$ / $\mathrm{s}$ ausbaubar ist. Zur Umsetzung dieses Vorhabens hat die TFH Wildau im November 1996 zusammen mit anderen Hochschul- und Forschungseinrichtungen Potsdams und Umgebung eine entsprechende Verwaltungsvereinbarung mit der Universität Potsdam abgeschlossen. Die Universität Potsdam installiert einen $34 \mathrm{MB} / \mathrm{s}$ B-Win-Anschluß und übernimmt die Verteilfunktion im MAN Potsdam im Sinne eines Brandenburgischen Forschungsnetzes. Auf der Grundlage einer Nutzungsvereinbarung wird die Energieversorgung Potsdam (EVP) die erforderliche Infrastruktur errichten. Gegenwärtig werden die Vorbereitungen zum Bau einer GlasfaserLeitungsverbindung zwischen Wildau und Potsdam getroffen. Ein Zugang zum Berliner Breitbandnetz (BRAIN) ist vorgesehen. Die TFH Wildau nimmt im Rahmen der MAN-Vereinbarung einen $2 \mathrm{MB} / \mathrm{s}$ B-WiN-Anschluß in Anspruch.

Durch diesen relativ kurzfristig zu realisierenden Anschluß an das B-WiN ist gegenüber dem jetzigen Stand eine Erhöhung der Bandbreite fuir den Internetzugang 
gegeben, wodurch sich kurze Reaktionszeiten bei der Inanspruchnahme von Internetdiensten erreichen lassen.

Langfristig ist die Teilnahme am CorporateNetwork von Forschungs- und Bildungseinrichtungen (Sprach- und Videouibertragung, Telefonie, Teleworking, Telelearning, Videokonferenzen) vorgesehen, die nur möglich ist uiber eine ATM-Anbindung an ein MetropolitanAreaNetwork (MAN). Die internen Voraussetzungen an der TFH Wildau wurden durch den 1. Abschnitt des Aufbaus des ATM-Campusnetzes geschaffen.

\section{Dienste}

Die Technische Fachhochschule Wildau ist uiber den Domaine-Namen „tfh-wildau.de“ weltweit erreichbar. Informationen ïber die TFH sind durch unseren WWWServer abrufbar. WWW steht für World-Wide-Web und ist die grafische Benutzeroberfläche im Internet. Die vollständige URL-Adresse der TFH Wildau lautet http:// www.tfh-wildau.de. Von der Homepage ausgehend können uiber sogenannte Links (Verweise) weitere Seiten aufgerufen werden, die über weitere Inhalte Auskunft geben. So sind beispielsweise Information uiber das Studienangebot, den Studienort Wildau, Ansprechpartner, Partner im Technologietransfer, aktuelle Informationen, wie Workshops usw. enthalten. Die Homepage der TFH Wildau ist im Bild 2 dargestellt.

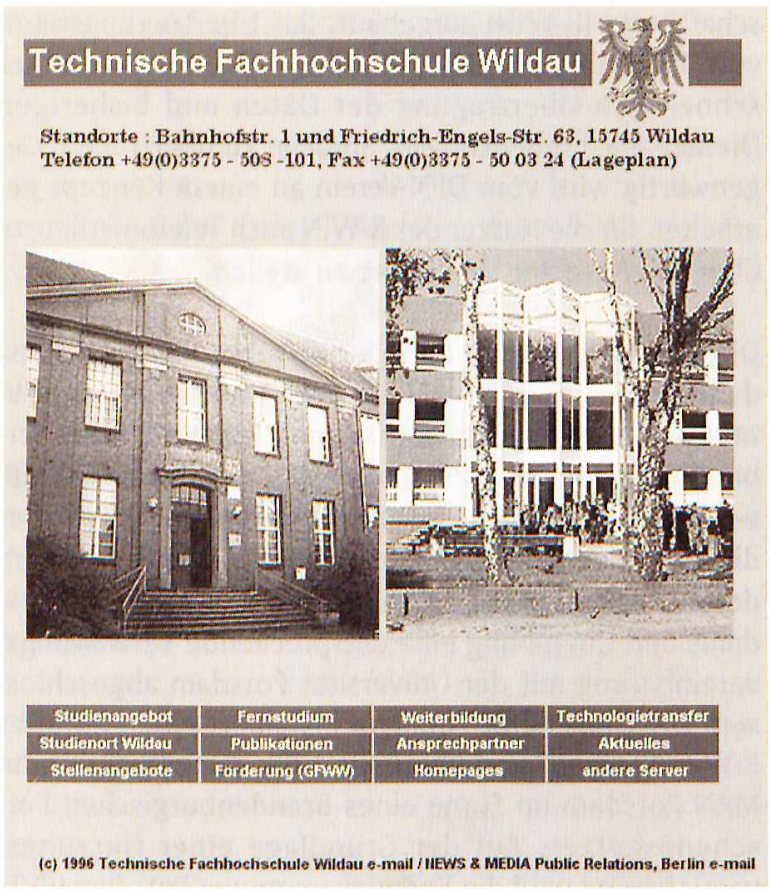

Bild 2: Homepage der TFH - Wildau

Seit April '97 besteht auch die Möglichkeit, daß jeder autorisierte Nutzer an der TFH seine eigene Homepage iiber unseren WWW-Server in das Netz stellt. Ein Link führt auf die entsprechende Seite, d. h. die Datei in seinem Homeverzeichnis. Damit kann die eigene Homepage gepflegt, d. h. aktualisiert werden und ist trotzdem für alle Internetnutzer erreichbar.
Neben der Nutzung von WWW hat sich die elektronische Post e-mail einen festen Platz erobert. Jeder autorisierte Nutzer an der TFH erhält automatisch seine email Adresse, die sich aus seinem Anmeldenamen, dem Fachbereichskïrzel und dem Domainenamen zusammensetzt. Der Vorteil dieses Dienstes gegenüber der "gelben Post" besteht neben des Null-Tarifs (für TFHStudenten und Mitarbeiter) darin, daß der Ansprechpartner diese Mail sofort von seinem Mail-Server abrufen und elektronisch weiterverarbeiten kann.

Neben WWW und e-mail sind noch weitere Dienste durch das Hochschulrechenzentrum verfuigbar bzw. werden aufgebaut.

\section{Zusammenfassung}

Durch den Anschluß der TFH an das MAN Potsdam wird sowohl ein leistungsfähiger Internetzugang, als auch eine Voraussetzung für die Teilnahme am CorporateNetwork von Forschungs- und Bildungseinrichtungen geschaffen.

Mit dem Aufbau des ATM LANs an der TFH Wildau entstand ein neues zeitgemäßes aber auch für die Zukunft weiter ausbaubares ATM-Netz.

Der weitere Aufbau des Netzes erfolgt stufenweise in Abhängigkeit von der Verfügbarkeit der finanziellen Mittel. Fachbereiche, die noch nicht an das ,große Netz“ angeschlossen sind, müssen mit provisorischen Lösungen auskommen (Modemverbindungen).

Die Bereitstellung von Diensten, Rechnerleistungen und Service im Rahmen der Möglichkeiten und Zuständigkeiten werden weiterhin die Aufgaben des Hochschulrechenzentrums sein und sollen mit dazu beitragen, die TFH Wildau zu einem attraktiven Studienort zu entwikkeln.

\section{Literatur}

/1/ Zeitschrift Pan Dacom, Ausgabe 30, 1996.

\section{Verfasser}

Dipl.-Ing. Günter Schubert

Technische Fachhochschule Wildau Hochschulrechenzentrum Tel. (03375) 508-186

Dipl.-Ing. Norbert Miersch Technische Fachhochschule Wildau Hochschulrechenzentrum Tel. (03375) 508-185 